\title{
Produção científica sobre psicoterapias breves no Brasil e demais países latino-americanos (1990-2000)
}

\author{
Neci Sena Ferreira \\ Universidade Federal do Tocantins \\ Elisa Medici Pizão Yoshida \\ Pontifícia Universidade Católica de Campinas
}

\begin{abstract}
Resumo
O objetivo deste estudo foi o de delinear um perfil da literatura científica sobre as psicoterapias breves no Brasil e demais países da América Latina, entre 1990 e 2000, através de levantamento realizado nas seguintes bases eletrônicas de dados: Lilacs, PsycINFO, Medline, Adolec, Scielo, MedCarib, Teses Brasileiras, PAHO e WHOLIS. Os 81 resumos obtidos foram analisados segundo as seguintes categorias: distribuição da produção, autoria, afiliação institucional dos autores, fonte de publicação, ano da publicação, idioma, suporte de publicação, população alvo, enfoque teórico, natureza do trabalho. Os resultados sugerem que: 1) as bases de dados eletrônicas constituem atualmente importante fonte de acesso à produção brasileira sobre as psicoterapias breves, não se podendo dizer o mesmo dos demais países latino americanos; 2) apesar da diferença numérica, verifica-se um perfil semelhante quanto às variáveis estudadas para o conjunto das produções.
\end{abstract}

Palavras-chave: psicoterapia breve; produção científica; psicoterapia de tempo limitado; bases de dados

\begin{abstract}
Scientific production on brief psychotherapies in Brazil and other Latin-American countries (1990-2000). The purpose of this study was to outline a profile of the scientific literature concerning brief psychotherapies in Brazil and other countries of Latin America, from 1990 to 2000, based on an electronic databases survey. Data obtained from Lilacs, PsycINFO, Medline, Adolec, Scielo, MedCarib, Brazilian Thesis, PAHO and WHOLIS resulted in 81 abstracts analyzed according to the: production distribution, authorship, affiliation, source, publication year, language, document type, population, theoretic approach and publication type. Results suggested that: 1) the electronic databases are currently important sources for accessing the Brazilian brief psychotherapy production, but not for the other Latin America countries; 2) despite the quantitative difference of both productions, a similar profile may be verified respecting many of the variables under study.
\end{abstract}

Keywords: brief psychotherapy; scientific production; limited time psychotherapy; data bases

$\mathrm{H}$ á uma extensa produção científica sobre psicoterapias breves (PBs) tanto na literatura estrangeira quanto na nacional, sem que pesquisas de revisão sistemática ofereçam uma visão de conjunto dessa área do conhecimento. Especialmente na última década, não se tem notícia de qualquer trabalho publicado que apresente um quadro geral da evolução dessas técnicas. Diante de tal realidade, julgouse oportuno proceder a um levantamento sobre as PBs no Brasil e demais países latino-americanos, no período de 1990 a 2000, procurando delinear um perfil de algumas tendências atuais e perspectivas científicas da área.

A opção pela produção brasileira sobre as PBs pautou-se não apenas no interesse das autoras pelo tema, mas também na relevância que uma visão de conjunto das características da produção nacional poderia ter para inúmeros profissionais que praticam e/ou pesquisam essa modalidade de psicoterapia em nosso meio. Por outro lado, considerou-se que o cotejamento com a produção latino-americana permitiria a ampliação deste quadro para além das fronteiras nacionais, mantendo, todavia, o contexto sócio-cultural compartilhado por países que, como o nosso, enfrentam limitações de ordem econômica e educacional, o que tem conseqüências diretas sobre a qualidade e a natureza da prática, da pesquisa e também da produção científica de uma determinada área do conhecimento.

Para a consecução desse objetivo, foram estabelecidas as seguintes categorias de análise: distribuição de freqüência, autoria, afiliação institucional dos autores, fontes de publicação, ano de publicação, idioma, suporte da publicação, população alvo, enfoque teórico e natureza do trabalho (relato de experiência clínica, trabalho teórico ou pesquisa).

Não se ignora que os países latino-americanos constituem um conjunto heterogêneo, seja no que tange aos aspectos sócio-culturais, seja no que se refere à qualidade da produção científica que detêm. No entanto, a escassez ou mes- 
mo a inexistência de referências bibliográficas da maioria deles na amostra obtida, mostrou que uma análise da produção de cada um dos países, era inviável. Sendo assim, a opção por reunir os trabalhos num único bloco, latino-americano, foi vista como a melhor maneira de se proceder a uma primeira abordagem desta produção, no que pese a artificialidade criada por este expediente.

A escolha do período de 1990 a 2000 - um espaço de tempo relativamente longo - justifica-se pelo fato de haver no Brasil poucos pesquisadores investigando as psicoterapias breves. Não se esperava encontrar, portanto, uma produção muito extensa num levantamento sistemático. Expectativa semelhante existia em relação aos demais países latino-americanos, por também não deterem tradição nessa área do conhecimento, a não ser por alguns poucos autores ou grupos de pesquisa existentes na Argentina, Uruguai e Chile.

Dentre os autores argentinos pode-se citar, por exemplo, Fiorini (1975/1989), Braier (1984/1986) e Kusnetzoff (1975/ 1980), todos com obras já traduzidas para o português nos anos 80 , e bastante conhecidos pelos brasileiros. Do Uruguai, o grupo mais conhecido é o do Instituto Agora (Defey, Elizalde, \& Rivera, 1995), e do Chile, o grupo de Santiago (De la Parra, 1993). Vale destacar, ainda, que o trabalho desses dois grupos encontra-se apresentado em livro recentemente publicado sobre as psicoterapias breves de orientação psicodinâmica, em nosso meio (Yoshida \& Enéas, 2004). O do grupo uruguaio em capítulo intitulado "Psicoterapia psicoanalítica focal del Instituto Agora (Montevideo)" (Defey, Elizalde, \& Rivera, 2004), e o do grupo chileno "Psicoterapia breve en el grupo de Santiago de Chile: la indicación adaptativa y el continuo "expressivo-de-apoyo"” (De la Parra, 2004).

O levantamento de bibliografia concernente a uma área geográfica de tal abrangência e de um período de tempo tão longo, impunha, naturalmente, limites que condicionavam as vias de acesso aos dados desejados. Ademais, havia necessidade de se garantir critérios mínimos de comparabilidade quanto às características investigadas. Nesta medida, o recurso às bases de dados internacionais mostrou-se como a via mais adequada para a pesquisa pretendida, já que todos os trabalhos nelas disponibilizados passam pelos mesmos critérios de indexação e se enquadram em padrões cientificamente desejáveis.

\section{Método}

\section{Material}

Foram considerados neste estudo trabalhos indexados nas seguintes bases de dados: 1. PsycINFO (American Psychological Association, http://www.apa.org); 2. MEDLINE, produzida pela National Library of Medicine (http://www.usp.br/sibi/biblioteca/medline.htm); 3. LILACS, Literatura Latino-Americana e do Caribe em Ciências da Saúde (http://www.bireme.br); 4. SciELO, Scientific Electronic Library Online (http://www.scielo.br); 5. TESES BRASILEI$R A S$, Sistema Teses Brasileiras (TB), (http://www.ibict.br); 6. $A D O L E C$, projeto da OPAS, relativo à adolescência, coorde- nado pelo Programa de Saúde Materno-Infantil (http:// www.adolec.br); 7. PAHO, Pan American Health Organization (http://bireme.br/iahlil/homepage); 8. WHOLIS, Sistema de Informação da Biblioteca da Organização Mundial de Saúde (http://www.bireme.br/iahlil/homepage.htm); 9. MEDCarib, coordenada pelo Centro Coordenador da Rede MedCarib, The Medical Library, University of the West Indies, Mona, Kingston-Jamaica (http://www.bireme.br/iahlil/ homepage.htm).

Juízes

As duas autoras atuaram como juízes dos itens 8 (Enfoque Teórico) e 9 (Natureza do Trabalho) avaliando, de forma independente, os resumos dos artigos. No procedimento de avaliação foram utilizadas categorias previamente estabelecidas.

\section{Procedimento}

Para levantar os resumos nas bases de dados em português, foram utilizadas as seguintes expressões booleanas: Psicoterapia and Breve e Psicoterapia and Tempo Limitado. Essas duas combinações foram utilizadas tendo em vista que na literatura internacional as psicoterapias de objetivos limitados (Knobel,1986) têm recebido essas duas denominações genéricas. Uma outra forma de denominação encontrada é Psicoterapia focal, mas esta costuma designar técnicas específicas de psicoterapias como, por exemplo, a técnica inicialmente defendida por Lemgruber (1984), ou a do grupo uruguaio já mencionado (Defey et al., 1995, 2004). Empregando-se o termo Psicoterapia de curta duração, uma outra forma de designação desta modalidade terapêutica, nenhum resumo foi localizado. Para pesquisar as bases de dados em inglês, usou-se a seguinte combinação de termos: (brief or short-term or limited time) and psychotherapy and Brazil.

Obtido o material, procedeu-se à leitura e seleção dos resumos para identificar aqueles que se enquadravam nos requisitos da pesquisa e os que se repetiam em mais de uma base de dados. Em seguida, os dados foram tabulados levando em consideração as seguintes categorias: (1) distribuição de frequiência; (2) autoria (única, dupla ou múltipla, para mais de dois autores); (3) afiliação institucional dos autores; (4) fonte de publicação; (5) ano da produção; (6) idioma; (7) suporte da produção (artigo; capítulo de livro; livro; dissertação de mestrado; tese de doutorado); (8) população-alvo (crianças; adolescentes; adultos; pacientes psiquiátricos ambulatoriais; pacientes especiais; dependentes químicos; alunos em situações específicas; profissionais em treinamento; grupos familiares e relacionamento paciente-terapeuta); (9) enfoque teórico; e (10) natureza do trabalho (relato de experiência clínica, trabalho teórico ou de pesquisa).

Em etapa prévia à análise e discussão dos resultados, estimou-se o índice de acordo entre os juízes, sobre as avaliações independentes relativas aos itens 8 (Enfoque Teórico) e 9 (Natureza do Trabalho), como forma de se certificar da fidedignidade dos julgamentos.

$\mathrm{Na}$ avaliação quanto ao Enfoque Teórico, foram utilizadas as seguintes categorias, previamente estabelecidas: a) PBs de orientação psicodinâmica ou tempo limitado; b) inter- 
venção breve ou psicoterapia breve (sem determinar o enfoque teórico); c) PBs associadas a outros enfoques teóricos.

Foram utilizadas as seguintes definições para a identificação da Natureza dos Trabalhos: (a) estudo empírico - pesquisa envolvendo levantamento e análise de dados com a finalidade de conhecer um fenômeno ou de testar uma hipótese; (b) relato de experiência - aplicação de novos programas ou formas de intervenção desenvolvidas empiricamente e que são descritos por seus autores e/ou praticantes; (c) revisão de literatura - revisão sistemática da literatura concernente a um tema ou tópico específico com o objetivo de delinear o estado da arte, identificar principais autores e/ ou pesquisas, ou estimar a eficiência de uma técnica; (d) trabalho teórico - voltado para a reflexão de um tema, tópico ou conceito teórico ou para a descrição de uma técnica psicoterapêutica; e) trabalho teórico ilustrado - voltado para a reflexão de um tema, tópico ou conceito teórico, acompanhado de um ou mais casos clínicos práticos com a finalidade de ilustração.

Obteve-se um índice kappa igual a 0,85 para a categoria Enfoque Teórico e 0,86 para a Natureza do Trabalho, índices considerados satisfatórios para a categoria dos dados. As demais variáveis, que não dependem de interpretação, foram tabuladas apenas pela primeira autora.

Para avaliação da média brasileira de publicações/ano excluiu-se uma referência da base LILACS que não continha indicação do ano de publicação e a única obtida de 2000 da Teses Brasileiras, em função da coleta de dados ter sido efetuada no início de 2001, quando nem todos os fascículos dos volumes de 2000 haviam sido indexados. Ou seja, no cálculo da média apenas foram considerados os registros de 10 anos, de forma a se ter um valor mais fidedigno da produção anual média.

\section{Resultados e discussão}

Da pesquisa realizada nas bases de dados LILACS, PsycINFO, MEDLINE, ADOLEC, SciELO, MedCaribe, Teses Brasileiras, PAHO e WHOLIS, obteve-se um total de 361 documentos dos quais, 280 referiam-se a publicações de outros tipos de trabalhos, isto é, que não tinham nenhuma relação com as psicoterapias breves e apareceram na pesquisa em virtude de seus resumos apresentarem as palavras breve, curta duração ou tempo limitado.

Identificada a produção específica sobre psicoterapias breves (PBs) em cada uma das bases de dados pesquisadas, foram encontrados os seguintes índices: LILACS, de um total de 183 referências, apenas $50(27,86 \%)$ relativas às $\mathrm{PBs}$; PsycINFO apresentou 46 referências, sendo 15 (32,50\%) de PBs; SciELO, com duas referências e Teses Brasileiras, com 13, apresentaram $100 \%$ de referências sobre PBs; e, o menor índice é da ADOLEC, com apenas um artigo, representando $2,60 \%$ do total de 38 referências ali encontradas. Não havia referências sobre PBs entre as 77 obtidas na MEDLINE, ou entre as duas da MedCaribe. Na PAHO e WHOLIS não foram obtidas referências sobre PBs. Resultando, portanto, num total de 81 referências, o que representa $22,43 \%$ do total dos documentos inicialmente levantados.
Das 50 referências encontradas na LILACS, 34 são de trabalhos brasileiros e 16 dos demais países da América Latina, o que faz com que ela detenha o maior percentual de publicações sobre PBs, tanto no Brasil como na América Latina, quando comparada às demais bases. Esse fato se justifica por ser a LILACS uma base de dados voltada para a divulgação da literatura científica produzida na América Latina e no Caribe na área de Ciências da Saúde e desde sua criação em 1982, vem ampliando significativamente o número de periódicos e outros documentos indexados.

Verifica-se igualmente nessa base, a superioridade evidente de referências brasileiras indexadas no período estudado $(68 \%)$, em relação ao conjunto dos demais países latinoamericanos (32\%).

A base de dados PsycINFO revelou apenas oito (53\%) trabalhos brasileiros e sete $(47 \%)$ de outros países da América Latina, uma inegável diferença em relação à LILACS. As freqüências relativas das duas produções aqui estariam mais equilibradas, não fosse o fato de que se está comparando a produção indexada de um país - Brasil - em relação a um grupo de países.

Em relação à MEDLINE, também uma base de dados internacional, apesar de apresentar um elevado número de publicações relativas à Psicologia de diversos países, só apresentou um periódico latino-americano da área, o Avances em Psicologia Clínica Latinoamericano, publicado pela Fundación para el Avance de la Psicologia, de Bogotá, Colômbia e que não publicou trabalhos relacionados ao tema. Da área biomédica, há os periódicos mais antigos dos países da região, posto que não foram incluídos nessa base, nos últimos 20 anos, novos títulos latino-americanos (Castro, Ferreira, \& Vidili, 1996). Todas as referências sobre as PBs, encontradas na MEDLINE, procediam dos países da América do Norte, principalmente dos Estados Unidos, e dos países da Europa.

Na ADOLEC, uma base de dados bibliográfica contendo trabalhos voltados para a adolescência, obteve-se apenas uma referência sobre PBs. Trata-se de trabalho realizado no Brasil: "Psicoterapia de grupo de tempo limitado (PGTL) com adolescentes", de Dal Fabbro, Horikoshi, Ishara e Contel, de 1993, publicado pela revista Medicina, da Faculdade de Medicina da USP de Ribeirão Preto.

Quanto à SciELO $(\mathrm{N}=2)$ e Teses Brasileiras $(\mathrm{N}=13)$, veiculam apenas a produção nacional, não se esperando, portanto, que apresentem literatura de outros países. Cabe, referir que enquanto a base de Teses Brasileiras reúne um acervo considerável de teses e dissertações, a SciELO é uma base bastante recente, tendo iniciado suas atividades a partir de 1997.

Excluindo-se essas duas últimas bases por veicularem apenas produção nacional, têm-se 43 referências brasileiras indexadas nas demais bases internacionais pesquisadas e 23 latino-americanas, o que corresponde, respectivamente, a $65 \%$ e $35 \%$ do total de referências acessadas, uma diferença estatisticamente significante $(p<0,02)$.

No entanto, uma maior proporção de trabalhos indexados não corresponde, necessariamente, a maior produção na área, já que nem toda ela chega a ser submetida à indexação pelos editores, seja por não atender aos critérios das bases de da- 
dos, ou porque não houve iniciativa ou possibilidade por parte dos editores para fazê-lo. Entretanto, um maior número de trabalhos indexados, confere maior visibilidade e acesso à produção em questão, com repercussões evidentes sobre o impacto desta sobre a área do conhecimento específica e sobre as disciplinas que com ela guardam algum tipo de interface. Vale aqui, também, a argumentação de que se está comparando a produção nacional à de um conjunto de países. E nesta medida, a presença mais significativa da produção brasileira nas bases internacionais, garante-lhe posição de destaque, quando comparada a cada um dos demais países latino-americanos.

Relativamente ao item Autoria, a autoria única representa mais da metade dos trabalhos acessados (51,93\%). Para autoria dupla, tem-se o índice de $28,40 \%$ e, para autoria múltipla, $19,75 \%$. Dentre os que tiveram autoria única, $13(40,47 \%)$ são relativos a dissertações e teses e, portanto, necessariamente de autores únicos. Os demais trabalhos de autoria única $(\mathrm{N}=25)$ aparecem numa freqüência muito próxima aos de autoria dupla $(\mathrm{N}=23)$ e múltipla $(\mathrm{N}=16)$, não havendo diferença significante entre eles $(p>0,25)$. Essa característica foi verificada tanto para a produção nacional, quanto para a dos demais países latino-americanos.

A distribuição dos trabalhos entre as diferentes modalidades de autoria, acompanha o movimento geral observado na literatura científica internacional, em que o trabalho de pesquisadores individuais vem gradativamente dando espaço a autorias duplas ou múltiplas, decorrentes do trabalho em colaboração com outros autores, que podem ser ou não da mesma instituição, e que, em alguns casos, são até de diferentes países. No Brasil, essa tendência tem ganhado corpo com a criação do Diretório de Grupos de Pesquisa do CNPq, outra fonte interessante de acesso à produção científica, que, todavia, não chegou a ser incluída na presente pesquisa.

No item Afiliação Institucional dos Autores Brasileiros, encontrou-se uma grande concentração de trabalhos sem qualquer indicação de afiliação. Do total de 58 referências acessadas, em 60,34\% delas não há informações sobre a ligação de seus autores às instituições. Também nas referências dos demais países da América Latina $(\mathrm{N}=23)$, o índice de trabalhos sem informação da Afiliação Institucional chega a $65,22 \%$. Nesse subitem, fica-se também sem a informação do país a que pertence(m) o(s) autor(es).

A ausência de informação sobre a instituição de origem nas bases de dados prejudica a pronta identificação da procedência de trabalhos de autores menos conhecidos na área, ao mesmo tempo em que dificulta o estabelecimento de ligação entre o autor e uma determinada instituição, ou ainda entre o autor e seu grupo de pesquisa. Especialmente nos dias atuais, em que, como dito acima, os grupos de pesquisa ganham importância na produção científica de todas as áreas do conhecimento, a afiliação institucional pode ser um dado relevante no processo de seleção de informação e no mapeamento dos centros de referência sobre o assunto pesquisado.

Com relação à distribuição geográfica das instituições brasileiras referidas, observou-se a supremacia daquelas situadas nos Estados do Sul e do Sudeste, que detêm a totalidade dos trabalhos, com destaque para o Estado de São Paulo, que res- ponde por $91,30 \%$ das publicações. Dentro do Estado de São Paulo, a Universidade de São Paulo (USP), com produções da Faculdade de Medicina de Ribeirão Preto $(\mathrm{N}=3)$ e do Instituto de Psicologia $(\mathrm{N}=11)$, aparece em primeiro lugar, seguida pela Pontifícia Universidade Católica de São Paulo (PUC-SP) $(\mathrm{N}=4)$, Universidade Estadual de Campinas (UNICAMP) $(\mathrm{N}=$ 2) e a Escola Paulista de Medicina $(\mathrm{N}=1)$. Também da Região Sudeste, mas do estado de Minas Gerais, tem-se a Universidade Federal de Uberlândia (UFU), com um trabalho.

Face ao número expressivo de referências sem indicação da afiliação institucional dos seus autores, não se pode concluir que essas instituições sejam as que realmente apresentam maior produção em PBs no Brasil. De toda forma, estes resultados alinham-se com os referidos por Yamamoto, Souza, e Yamamoto (1999), quando analisaram a produção científica em Psicologia através dos periódicos brasileiros no período de 1990 a 1997, e concluíram que a grande maioria dos artigos publicados nos periódicos pesquisados se originou da Região Sudeste, com participação também da Região Sul.

Também para os demais países latino-americanos, apenas uma pequena percentagem das publicações apresenta a Afiliação Institucional dos seus Autores $(\mathrm{N}=8)$. São três instituições chilenas - Instituto de Psiquiatría Dr. José Horwitz Barak $(\mathrm{N}=4)$, Sociedad Chileña de Psicologia Clínica $(\mathrm{N}=1)$ e Unidade de Psicoterapia Breve, do Hospital Salvador de Santiago $(\mathrm{N}=1)$ - e uma mexicana, o National Institute of Perinatology, da Cidade do México $(\mathrm{N}=2)$.

Como no caso da produção brasileira, esses dados devem ser interpretados com cautela, já que não se conhece a afiliação institucional da maioria dos autores e se sabe que outros países como, por exemplo, a Argentina e o Uruguai, acima mencionados, detêm produção na área.

Especificamente na Argentina, pioneira na América Latina no campo das psicoterapias breves, sua produção científica em saúde mental, nos anos 70 e 80, apresentava um cunho eminentemente social, derivado da prática e da investigação no ambiente institucional. Com as crises político-financeiras e sociais que se sucederam naquele país na última década, é razoável supor que esta área tenha sido afetada, e sua produção científica comprometida.

Quanto aos demais países, a falta de tradição na área das psicoterapias breves, aliada à escassez de dados mais objetivos quanto às condições sócio-político-institucionais que balizam a prática dos pesquisadores em cada um deles, inviabilizam uma análise mais profunda sobre eventuais motivos para ausência de referência à afiliação institucional observadas.

Em face das limitações enfrentadas e tomando como base a amostra total, pode-se apenas afirmar, que nela se encontra identificada uma pequena parte dos autores de instituições brasileiras, chilenas e mexicanas; nada se podendo dizer sobre a afiliação institucional dos autores dos demais países latino-americanos.

Relativamente ao item Fonte de Publicação (Tabela 1), um dado interessante é observar o grande número de periódicos da área médica que focalizam as PBs. Destacando-se o Jornal Brasileiro de Psiquiatria, o periódico brasileiro com maior número de referências $(\mathrm{N}=13)$. 
Tabela 1

Distribuição das referências segundo o periódico e a instituição editora no Brasil

\begin{tabular}{llcc}
\hline \multicolumn{1}{c}{ Título do periódico } & \multicolumn{1}{c}{ Instituição / Editora } & $\mathrm{f}$ & $\%$ \\
\hline Arquivos Brasileiros de Medicina (Rio de Janeiro-RJ) & Ed.Cient.Nacional & 1 & 1,72 \\
Boletim de Psicologia (São Paulo-SP) & Soc.Psic.S.P. & 4 & 6,90 \\
Contexto (São Paulo) & Sem Resumo & 1 & 1,72 \\
Jornal Brasileiro de Ginecologia (Rio de Janeiro-RJ) & UFRJ & 1 & 1,72 \\
Jornal Brasileiro de Psiquiatria (Rio de Janeiro-RJ) & UFRJ & 13 & 22,41 \\
Medicina - Ribeirão Preto (Ribeirão Preto-SP) & FM-HC-USP(RP) & 3 & 5,17 \\
Psicologia: Reflexão e Crítica (Porto Alegre-RS) & UFRGS & 3 & 5,17 \\
Psycotherapy and Psychosomatics (Suíça) & S. Karger & 1 & 1,72 \\
RBM-Revista Brasileira de Medicina (Ribeirão Preto-SP) & Grupo Ed.Moreira Jr. & 2 & 3,45 \\
Revista Brasileira de Psiquiatria (São Paulo-SP) & Assoc.Bras.Psiq. & 2 & 3,45 \\
Revista da ABP-APAL (São Paulo-SP) & Assoc.Bras.Psiq. & 4 & 6,90 \\
Revista de Psiquiatria Clínica (São Paulo-SP) & FM-USP & 1 & 1,72 \\
Revista Estudos de Psicologia (Campinas-SP) & PUCCAMP & 1 & 1,72 \\
Revista HCPA \& FM-UFRGS (Porto Alegre-RS) & UFRGS & 1 & 1,72 \\
RJ-Laborterápica Bristol (Rio de Janeiro-RJ) & Lab. Bristol & 1 & 1,72 \\
Temas (São Paulo-SP) & Hosp.Serv.Publ. & 1 & 1,72 \\
Temas sobre Desenvolvimento (São Paulo, SP) & Memnon Ed.Científica & 1 & 1,72 \\
Não-publicados (Teses e Dissertações) & & 17 & 29,31 \\
\hline Total & & 58 & 100,00 \\
\hline
\end{tabular}

Dentre os periódicos médicos, há ainda: a Revista Brasileira de Psiquiatria, Revista de Psiquiatria Clínica (SP), Revista da ABP-APAL (SP), Revista HCPA \& FM-UFRGS, RBM Revista Brasileira de Medicina (Ribeirão Preto), RJLaborterápica Bristol (RJ), Jornal Brasileiro de Ginecologia (RJ) e Arquivos Brasileiros de Medicina (RJ).

A verificação destes elevados índices de divulgação de trabalhos sobre as PBs em jornais e revistas médicas sugere a relevância que elas têm enquanto objeto de pesquisa junto a essa categoria profissional. Exemplos que apontam para esta tendência podem ser confirmados pelas seguintes referências observadas na amostra: Knobel (1991), Lemgruber (1993), Schoueri \& Segre (1999), Lowenkron (1990), Eizirik $(1991,1993)$ e muitos outros. Todos eles destacados pesquisadores das psicoterapias breves em nosso meio.
Observamos, ainda, que o único trabalho de autores brasileiros a figurar em um periódico internacional, é da área médica. Trata-se do artigo de Eizirik et al. (1991), intitulado "Observing countertransference in brief psychotherapy" publicado no Psycotherapy and Psychosomatics, periódico editado na Suíça.

Dentre os periódicos da psicologia destacam-se: o Boletim de Psicologia, da Sociedade de Psicologia de São Paulo $(\mathrm{N}=4)$, Psicologia: Reflexão e Crítica, da UFRGS $(\mathrm{N}=3)$ e Estudos de Psicologia (PUC-Campinas) $(\mathrm{N}=1)$.

Conforme observado na produção brasileira, também para os outros países da América Latina há um predomínio de publicações sobre as PBs em periódicos ligados à Psiquiatria (ver Tabela 2). De la Parra, também psiquiatra, é o autor chileno que apresentou maior produção nas bases de dados pesquisadas (De la Parra, 1993; De la Parra, Boetsch, \& Ruimalló, 1995).

Tabela 2

Distribuição das referências segundo o periódico e a instituição editora dos demais países da América Latina

\begin{tabular}{llrr}
\hline \multicolumn{1}{c}{ Título do periódico } & \multicolumn{1}{c}{ Instituição / Editora } & \multicolumn{1}{c}{$\mathrm{f}$} & \multicolumn{1}{c}{$\%$} \\
\hline Archivos Venezolanos Psiquiatria e Neurologia (Venezuela) & Soc.V. Psiq. & 1 & 4,35 \\
Coletivo Luchas Contra la Violencia de las Mujeres (México) & Sem indicação & 1 & 4,35 \\
Niños-Revista de Neuropsiquiatria Infantil (Venezuela) & Minist.Sanidad y Ass. & 1 & 4,35 \\
Revista Chilena Neuro-Psiquiatria (Chile) & Soc.Chil.Neurol. & 5 & 21,74 \\
Revista Chileña Psicoanalisis (Santiado do Chile) & Assoc.Psc.Chile & 1 & 4,35 \\
Revista de Psiquiatria (Santiago do Chile) & Soc.C.Salud M. & 5 & 21,74 \\
Revista Interamericana de Psicologia (Venezuela) & Soc.Interam.Psic. & 1 & 4,35 \\
Revista Medica del Cobre (Chile) & Serv.Med.Corp.Nac. & 2 & 8,70 \\
Revista Mexicana Psicologia (México) & Sem indicação & 3 & 13,04 \\
Revista Sanidad Medica (México) & Sem indicação & 1 & 4,35 \\
Salud Mental (México) & Sem indicação & 1 & 4,35 \\
Terapia Psicologica & Soc.Chilena Psiq. & 1 & 4,35 \\
\hline Total & & 23 & 100,00 \\
\hline
\end{tabular}


A prevalência de autores da área médica é uma tendência verificada desde as origens das psicoterapias breves, nos anos 1950, quando psiquiatras que trabalhavam em clínicas públicas ou hospitais gerais procuravam responder ao crescimento da demanda por psicoterapias. Esse, por exemplo, foi o caso dos pioneiros, Balint (1966) e Malan (1963, 1976/1981, 1979/1983), na Clínica Tavistock, em Londres; de Sifneos (1972, 1979/1989, 1984) no Hospital Geral de Massachussetts; de Davanloo (1979, 1980), no Hospital Geral de Montreal; ou ainda, de Mann (1973) no Departamento de Psiquiatria da Faculdade de Medicina de Boston. Todos eles, com formação psicanalítica, defrontaram-se com o problema das longas filas de espera existentes e buscaram, na delimitação da situação- problema, uma forma de prestar auxílio eficiente ao maior número possível de pessoas (Yoshida, 1990). Essa tendência se repete na segunda geração de autores internacionais sobre as psicoterapias breves (por exemplo: Luborsky, 1984), assim como, da terceira geração (por exemplo: Crits-Christoph \& Barber, 1991; Horowitz, 1990), conforme sugere a revisão apresentada por Yoshida (2004).

No Brasil, as psicoterapias breves foram abraçadas tanto por psicólogos quanto por médicos, desde os anos 1970. O predomínio de referências em periódicos médicos, quando comparadas às da psicologia, talvez seja o reflexo de haver mais revistas médicas indexadas nas bases de dados no período estudado do que, efetivamente, um maior número de produção médica. Pesquisas futuras de acompanhamento dessa produção deverão, no entanto, elucidar melhor essa questão. Por ora, registram-se aqui alguns trabalhos pioneiros, procurando justificar a afirmação acima de que, no Brasil, as PBs foram objeto de prática e de pesquisa por médicos e psicólogos, desde o início.

Efetivamente, os primeiros trabalhos de que se tem notícia são os de Hess e Meira (1972) e o de Oliveira, Pereira, e Bastos (1974), que relatavam suas experiências com atendimentos de tempo limitado. No primeiro caso, tratava-se da apresentação do Help, um programa da Sociedade de Psicologia do Rio Grande do Sul, que visava ao atendimento de jovens (de 12 a 18 anos), por psicólogos voluntários que, em sistema de plantão psicológico, procuravam fornecer ajuda "socialmente orientada". No segundo, apresenta-se a experiência realizada com dois grupos de psicoterapia, no Centro de Estudos do Instituto de Psiquiatria da UFRJ, buscando aplicar ali a técnica desenvolvida por Malan, em substituição à análise de grupo até então praticada, e que se verificava incompatível com a realidade institucional vivida. Apesar dessas iniciativas, foi somente nos anos 1980 que as psicoterapias breves passaram a ser mais claramente definidas e pesquisadas, em nosso meio, podendo-se então falar em uma "primeira geração de autores", representada por Knobel, Simon, e Lemgruber (Yoshida, 2004).

$\mathrm{O}$ interesse dos psicólogos pela pesquisa sobre PBs está evidenciado na proporção de teses e dissertações que correspondem a $29,31 \%$ do total da produção levantada nesta pesquisa. Desse total, a maior concentração está na área da Psicologia (86,66\%), ficando uma pequena parcela ligada à Psiquiatria $(13,34 \%)$. Isso demonstra que os psicólogos tam- bém pesquisaram sobre o tema ao longo dos anos 1990, mas não chegaram a publicar seus trabalhos, ou se o fizeram, priorizaram periódicos não-indexados, capítulos de livros ou livros. A título de exemplo, podem ser citados os livros Luto materno e psicoterapia breve, de Neli Klix Freitas (2000). e Psicoterapia breve dinâmica com pacientes borderline: uma proposta viável, de Rita Aparecida Romaro (2000), os quais resultaram das teses de doutorado de suas autoras, respectivamente de 1997 e 1999. Além desses, há ainda a tese de doutorado de Rita de Cássia Gandini (1995), cujos resultados foram parcialmente publicados em capítulo de sua autoria, "Psicoterapia breve em oncologia na mastologia", como parte do livro de Segre (1997).

Tomada ano a ano, a produção no Brasil apresentou um maior número de publicações no ano de 1999, com 12 referências, seguido por 1990 e 1993, cada um com sete. Quanto aos anos de menor produção, destacaram-se 1992 e 1996, ambos com apenas três referências indexadas. A média brasileira de publicações/ano ficou em torno de 5,6 e $D P=1,07$.

Com base nesses dados, pode-se dizer que a produção de PBs se manteve relativamente estável ao longo da década, tendo apresentado apenas uma elevação no ano de 1999. Justifica-se esta ampliação pelo maior número de títulos indexados na base de dados LILACS, que passou de quatro para nove periódicos, proporcionando maior visibilidade à literatura psicológica publicada nas revistas científicas brasileiras (Sampaio \& Peixoto, 2000).

Os maiores índices de divulgação da produção nos demais países da América Latina, foram alcançados em 1992, 1993 e 1995, com cinco trabalhos apresentados em cada um. No ano de 1990 foram publicados três trabalhos e em 1991, 1994, 1996, 1997 e 1998 apenas um trabalho em cada ano. Por ocasião da coleta de dados, não foi contado nenhum trabalho dos anos de 1999 e 2000 . A média de publicação/ano ficou em torno de 2,3 e o $D P=1,25$, excluindo-se o ano de 2000, como no cálculo da produção brasileira.

A diferença estatisticamente significante, entre a média de publicações/ano do Brasil com os demais países latino-americanos $(p<0,01)$, corrobora observações anteriores relativas à maior representatividade da produção brasileira não apenas em relação ao conjunto de países latino-americanos, como também em relação a cada um deles individualmente.

Como resultado da prevalência da produção brasileira, o português predominou enquanto idioma utilizado nos resumos, aparecendo em $61,73 \%$ dos casos. O espanhol foi o segundo idioma mais utilizado, com $19,75 \%$ das referências, e o inglês respondeu por $18,52 \%$ do total. Os resumos em inglês, são todos provenientes da PsycINFO, que apresenta os resumos em inglês, ainda que o trabalho completo original tenha sido publicado em outra língua, conforme indicado na categoria idioma (Language) dessa base de dados.

Em relação aos tipos de suporte das publicações brasileiras, do total de 58 documentos, destacaram-se os artigos em periódicos, com 68,97\%; teses, com 17,14\%; e dissertações, com 12,07\%. Uma referência, por não apresentar o resumo, impossibilitou a sua classificação quanto a este item. 
O predomínio de artigos de periódicos reflete o bom desenvolvimento do conhecimento produzido na área, na medida em que passam pela avaliação de membros do corpo editorial e/ou de consultores ad hoc, e são, portanto, referendados por outros especialistas que não apenas o(s) seu(s) autor(es). Especialmente quando se trata de artigos em periódicos indexados, como os constantes desta pesquisa, o controle da qualidade da produção é mais garantido. Além disso, trata-se de informações atualizadas, já que a periodicidade é outra exigência das bases de dados para aceitar e manter o periódico indexado. E, finalmente, há o fato de os periódicos quase sempre serem distribuídos para as bibliotecas das universidades brasileiras, além de contarem com um público assinante que garante melhor divulgação da informação entre os especialistas e pesquisadores da área.

Particularmente, em relação às dissertações e teses, embora também sejam submetidas à "avaliação por pares", representados pelos membros das bancas, costumam ficar restritas a uns poucos exemplares, dificultando o acesso às informações ali constantes. Essa realidade, embora esteja começando a se modificar com a popularização da Internet e a disponibilidade de trabalhos on-line para quaisquer interessados em sites mantidos pelo autor ou por sua instituição, ainda se encontra restrita a poucas iniciativas. Porém, representa uma possibilidade de melhor aproveitamento de trabalhos que antes somente podiam ser consultados nas bibliotecas das instituições em que foram defendidos.

A despeito de toda a popularidade que a Internet possa vir a ter, deve-se ressaltar que todo trabalho de conclusão de mestrado e doutorado deveria ser convertido em artigo e encaminhado para periódico indexado, possibilitando desta forma maior visibilidade ao conhecimento produzido. $\mathrm{O}$ artigo é uma modalidade sintética de transmissão da informação, característica relevante, quando se considera a quantidade cada vez maior de informações a serem absorvidas pelos especialistas de uma dada área do saber (Matoso \& Cecatti, 2002).

Mesmo sendo considerado um tipo de suporte popular, especialmente na nossa realidade acadêmica, os livros ou capítulos de livros precisariam, também, passar por peer review (avaliação de pares) para que se tivesse mais garantia da qualidade das informações por eles veiculadas. No entanto, só recentemente essa prática começou a ser utilizada, especialmente pelas agências de fomento, como a FAPESP, que submete a pareceristas ad hoc os livros por ela financiados. A falta de controle adequado da qualidade da informação veiculada em livros é, possivelmente, um dos motivos que levam os responsáveis pelas bases de dados brasileiras a ainda não contarem com a indexação deste tipo de suporte.

No que diz respeito à produtividade em PBs dos outros países da América Latina, o artigo em periódico representou a totalidade $(\mathrm{N}=23)$ das referências levantadas, não se tendo conhecimento de bases de dados específicas para dissertações de mestrado e teses de doutorado. No entanto, é possível que, tal como no Brasil, uma parte significante da produção sobre as PBs também seja produto de pesquisa acadêmica, não chegando todavia a ser publicada. Com relação aos livros e capítulos de livros, também não aparecem nas bases pesquisadas.
Relativamente à População-alvo dos trabalhos brasileiros, as categorias que mereceram maior número de pesquisas foram: pacientes psiquiátricos tratados em ambulatórios $(25,86 \%)$; pacientes especiais $(17,24 \%)$ - abrangendo: mulheres grávidas, com psicose puerperal, mastectomizadas, enlutadas; doentes renais crônicos; deficientes físicos; doentes com distúrbios crânio mandibular; doentes com disfunções sexuais; e profissionais em treinamento $(13,79 \%)$ - categoria que engloba: médicos, psicólogos, professores e supervisores durante estágios, residência médica em Psiquiatria ou cursos de pós-graduação. Em uma parcela significativa desses trabalhos $(12,07 \%)$, não foi possível o enquadramento em nenhuma das categorias criadas devido à falta de informações nos resumos e, em um trabalho (1,72\%), omitiu-se totalmente o resumo.

Com relação à população-alvo dos trabalhos realizados nos demais países da América Latina, o maior destaque foi para a categoria de pacientes especiais, que inclui: mulheres e crianças vítimas de violência, mulheres grávidas, pacientes com cefaléia, pacientes renais crônicos e alcoolistas, representando $30,43 \%$ do total. Também aqui os profissionais em treinamento e aperfeiçoamento de suas práticas clínicas mereceram a atenção em $21,74 \%$ dos trabalhos. Nesta subamostra, os trabalhos em que não foi possível definir a população-alvo chegaram a $30,43 \%$.

Tomando em conjunto os dados brasileiros e os dos outros países da América Latina, observa-se um maior número de trabalhos voltados para pacientes ambulatoriais, seja da saúde mental seja de outras clínicas médicas. Conforme já referido, as PBs surgiram como forma pragmática de resposta à grande demanda por atendimento institucional, mantendose dessa forma até os dias atuais. Além disso, é nesse contexto que as pesquisas são usualmente realizadas - e não nos consultórios particulares - ainda que muitos profissionais utilizem PBs também com essa população. Efetivamente, o ambiente institucional facilita a realização de pesquisas, pois, usualmente, torna mais fácil o acesso a amostras numerosas e mais homogêneas, constituindo-se no "laboratório" do pesquisador de saúde mental. Em que pese, portanto, o "perfil médico" dos periódicos, acredita-se que o predomínio de trabalhos contemplando populações, dos aqui chamados pacientes especiais, constitui antes a regra do que a exceção.

Além disso, nos dois segmentos estudados, há um número expressivo de trabalhos voltados para profissionais em treinamento, o que revela a preocupação dos pesquisadores com a formação de futuros terapeutas nesta modalidade de atendimento.

Em relação ao alto percentual de resumos em que não se conseguiu identificar a população-alvo, trata-se, na maioria, de trabalhos teóricos que não contemplam um tipo específico de população, mas que se destinam à discussão de princípios gerais das PBs.

$\mathrm{Na}$ comparação entre os dados brasileiros e os dos outros países da América Latina, em relação ao Enfoque Teórico, verificou-se uma equivalência entre todas as categorias. Para os trabalhos de orientação psicodinâmica, o percentual dos brasileiros foi de $41,38 \%$ e o dos demais países latino- 
americanos foi de 43,48\%. Quanto aos trabalhos de PBs que não determinaram o enfoque teórico, o Brasil apresentou $32,76 \%$ e os outros países da América Latina, 21,74\%; aqueles associados a outros enfoques teóricos representaram $22,41 \%$ no Brasil contra 26,09\% nos outros países latino-americanos. Em quatro trabalhos apresentados (dois brasileiros e dois dos outros países da América Latina) não foi possível definir o tipo de enfoque teórico adotado.

Diante desses dados, e apesar do percentual de trabalhos em que não se deixou explícito o enfoque teórico adotado $(29,63 \%)$, pode-se afirmar que as PBs de orientação psicodinâmicas representam uma forte tendência nos países latino-americanos, mantendo-se em conformidade com as origens desta modalidade terapêutica, que teve nos primeiros trabalhos de Freud sua inspiração (Malan, 1976/1981).

$\mathrm{Na}$ análise das referências brasileiras, quanto à Natureza dos trabalhos, observou-se uma maior incidência de estudos empíricos $(36,20 \%)$, seguida do relato de experiência $(24,13 \%)$, trabalhos teóricos $(12,06 \%)$, trabalhos teóricos ilustrados $(10,34 \%)$, e revisão de literatura $(6,90 \%)$. Em seis referências não foi possível definir o tipo de trabalho, devido à falta de resumo ou de informações esclarecedoras sobre esse item. $\mathrm{Na}$ produção dos demais países da América Latina, mais uma vez observam-se índices semelhantes aos brasileiros: os estudos empíricos representam 30,43\% e os relatos de experiências, 21,74\%; os estudos teóricos e os teóricos ilustrados aparecem cada um com percentual de $17,39 \%$ da produção indexada.

Verifica-se, portanto, equivalência quanto à natureza da produção brasileira e latino-americana, com maior número de estudos empíricos, o que vem ao encontro da tendência mais geral das ciências modernas, fortemente calcadas em evidências.

Para os trabalhos classificados como estudo empírico, as autoras definiram o tipo de delineamento de pesquisa empregado. Aqui merece menção o fato de que, do total de 28 trabalhos, dois terços correspondem a pesquisas brasileiras e apenas sete são provenientes de outros países. Ou seja, aqui também se verifica a prevalência da produção brasileira presente nas bases consultadas.

Quanto ao tipo de pesquisa realizada no Brasil, verificou-se elevada concentração de tipo quase-experimental que representou $61,90 \%$ e apenas $4,76 \%$ de tipo experimental. Quanto às de tipo descritivo, há 14,29\% de levantamento, 9,52\% de delineamento correlacional e 4,76\% de estudo de caso intensivo; restou um trabalho cuja classificação não foi possível. Em relação aos demais países da América Latina, predominaram as pesquisas de tipo quase-experimental $(71,43 \%)$, seguidas de delineamento correlacional $(28,57 \%)$, não se registrando os demais tipos de pesquisa.

Apesar da diferença entre as duas realidades, predominam pesquisas em que se busca certo controle das variáveis, de forma a garantir maior validade para os resultados encontrados. Ou seja, o elevado índice de pesquisas do tipo quaseexperimental vem confirmar ser esse o tipo de delineamento característico das Ciências Humanas e Sociais, em que os resultados são expressos em médias e significâncias estatísticas, porém, sem o rigor da randomização dos sujeitos e permitindo maior generalização dos resultados.

\section{Considerações finais}

A pesquisa realizada indicou um quadro bastante heterogêneo quanto à distribuição da produção científica indexada em bases de dados, sobre as PBs, na década de 1990. Verificou-se um desequilíbrio, tanto no âmbito interno - em que a produção é basicamente proveniente das regiões sudeste e sul do país - quanto no externo, com a presença significantemente maior de trabalhos brasileiros, em relação aos dos demais países latino-americanos.

A delimitação imposta pelos critérios adotados pelas bases de dados pode ser observada na semelhança qualitativa da produção sobre as psicoterapias breve, tanto nacional quanto dos demais países latino-americanos, com um perfil comum. Este pode ser descrito de forma sucinta como sendo caracterizado predominantemente por trabalhos publicados em periódicos, de autoria múltipla, relativos a pesquisas empíricas, segundo o delineamento quase-experimental ou correlacional, focalizando grupos específicos de pacientes ambulatoriais, ou de pessoas submetidas a condições sociais particulares, como são as pessoas vítimas de violência ou pertencentes a populações consideradas de risco.

Com isso, não se quer dizer, naturalmente, que não haja espaço para outros tipos de produção científica. Contudo, o delineamento do perfil acima, ajuda a destacar o papel do modelo hegemônico da ciência na atualidade e que se reflete nos critérios de avaliação adotados pelas bases de dados.

Frente às considerações acima, é possível concluir que o perfil traçado da produção sobre psicoterapias breves não é necessariamente generalizável para o restante da produção da área, já que não se têm dados para avaliar até que ponto esta parcela se alinharia com os critérios de seleção adotados pelas instituições gestoras para indexação. Deve-se considerar, entretanto, que a produção disponibilizada nas bases de dados conta, efetivamente, com maior visibilidade e, portanto, maior probabilidade de exercer impacto sobre os rumos do conhecimento na área. Nessa medida, pode-se dizer que as bases de dados eletrônicas constituem, atualmente, importante fonte de acesso para a produção brasileira sobre as psicoterapias breves, não se podendo dizer o mesmo para os demais países latino-americanos.

\section{Referências}

Balint, M., \& Balint. E., (1966). Técnicas Psicoterápicas en Medicina. México: Siglo XXI.

Braier, E. A. (1986). Psicoterapia breve de orientação psicanalítica (Trad. IPEPLAN). São Paulo: Martins Fontes. (Original publicado em 1984)

Castro, R. C. F., Ferreira, M. C. G., \& Vidili, A. L. (1996). Periódicos latinoamericanos: avaliação das características formais e sua relação com a qualidade cientifica. Ciência da Informação, 25(3), 357-367.

Crits-Christoph, P., \& Barber, J. P. (1991). Handbook of short-term dynamic psychotherapy. Nova York: Basic Books.

Davanloo, H. (1979). Technique of short-term psychotherapy. Psychiatric Clinics of North America, 2, 11-22.

Davanloo, H. (Org.). (1980). Short-term dynamic psychotherapy. Northvale: Jason Aronson.

Defey, D., Elizalde, H., \& Rivera, J. (Orgs.) (1995). Psicoterapia focal. Montevideo: Roca Viva. 
Defey, D., Elizalde, H., \& Rivera, J. (2004). Psicoterapia psicoanalitica focal del Instituto Agora (Montevideo). In E. M. P. Yoshida \& M. L. E. Enéas (Orgs.), Psicoterapias psicodinâmicas breves: propostas atuais (pp. 131166). Campinas: Editora Alínea.

De la Parra, G. C. (1993). El manual de psicoterapia dinámica de tiempo limitado: una nueva alternativa para entrenar terapeutas en un consultorio externo. Revista Chilena de Neuropsiquiatria, 31(4), 379-387.

De la Parra, G. C. (2004). Psicoterapia breve en el grupo de Santiago de Chile: la indicación adaptativa y el continuo "expresivo-de-apoyo". In E. M. P. Yoshida \& M. L. E. Enéas (Orgs.), Psicoterapias psicodinâmicas breves: propostas atuais (pp. 95-130). Campinas: Editora Alínea.

De la Parra, G. C., Boetsch, M. E., \& Ruimalló, P. (1995). Proyecto de investigación de la unidad de psicoterapia breve del Inst. Psiquiátrico Dr. José Horwitz Barak: primeiros resultados. Revista de Psiquiatria (Santiago do Chile), 12(3/4), 126-133.

Eizirik, C. L. (1993). Uma amostra de psiquiatria do Rio Grande do Sul e São Paulo: perfil demográfico e prática de consultório. Revista ABP-APAL, 15(3), 82-86.

Fiorini, H. J. (1989). Teorias e técnicas de psicoterapias ( $8^{\mathrm{a}}$ ed., C. Sussekind, Trad.) Rio de Janeiro: Francisco Alves. (Texto original publicado em 1975)

Freitas, N. K. (1997). O processo do luto materno através da psicoterapia breve. Tese de doutorado não-publicada, Pontifícia Universidade Católica de São Paulo, São Paulo.

Freitas, N. K. (2000). Luto materno e psicoterapia breve. São Paulo: Summus.

Gandini, R. C. (1995). Câncer de mama: evolução da eficácia adaptativa em mulheres mastectomizadas. Tese de doutorado não-publicada, Universidade de São Paulo, São Paulo.

Gandini, R. C. (1997). Psicoterapia breve em oncologia na mastologia. In C. D. Segre (Org.), Psicoterapia breve (pp. 215-235). São Paulo: Lemos.

Hess, H. D., \& Meira, L. A. (1972). HELP, Programa de ajuda psicológica à juventude. Psico, 4, 29-41.

Horowitz, M. (1990). Introdução à psicodinâmica: uma nova síntese (R. E. Starosta, Trad.). Porto Alegre, Artes Médica.

Knobel, M. (1986). Psicoterapia breve. São Paulo: EPU.

Knobel, M. (1991). Psicoterapia, psicoterapia breve e psicoterapia de emergência. Temas (São Paulo), 21(40/41), 193-201.

Kusnetzoff, J. C. (1980). Psicanálise e psicoterapia breve na adolescência (P. M. E. Cenacchi, Trad.). Rio de Janeiro: Zahar. (Texto original publicado em 1975)

Lemgruber, V. B. (1984). Psicoterapia breve a técnica focal. Porto Alegre: Artes Médicas.

Lemgruber, V. B. (1993). Da neurose de ansiedade ao distúrbio de ansiedade generalizada. Jornal Brasileiro de Psiquiatria, 42( 2 ), 111-115.

Lowenkron, T. S., \& Cheniaux , E., (1990). O ensino da psicoterapia psicanálitica: a indicação e o relato de uma terapia de tempo breve. Jornal Brasileiro de Psiquiatria, 39(5), 267-271.
Luborsky, L. (1984). Principles of psychoanalytic psychotherapy: a manual for supportive-expressive treatment. Nova York: Basic Books.

Malan, D. H. (1963). A study of brief psychotherapy. Londres: Tavistock.

Malan, D. H. (1981). As fronteiras da psicoterapia breve. Um exemplo da convergência entre pesquisa e prática médica (L. Knijnik \& M. E. Z. Schestatsky, Trad.). Porto Alegre: Artes Médicas. (texto original publicado em 1976)

Malan, D. H. (1983). Psicoterapia individual e a ciência da psicodinâmica (M. C. Juchem, Trad.). Porto Alegre: Artes Médicas. (Texto original publicado em 1979)

Mann, J. (1973). Time-limited psychotherapy. Cambridge, Massachusetts: Harvard University Press.

Matoso, M. C., \& Cecatti, J. G. (2002). O artigo científico na área da saúde: evolução, perspectivas e desafios. Revista de Ciências Médicas, 11(1), 3-5.

Oliveira, W. I., Pereira, W. L., \& Bastos, J. C. (1974). Grupo de psicoterapia breve. Jornal Brasileiro de Psiquiatria, 23(3-4), 377-392.

Romaro, R. A. (1999). Variação da eficácia adaptativa de pacientes borderline em psicoterapia breve dinâmica. Tese de doutorado não-publicada, Universidade de São Paulo, São Paulo.

Romaro, R. A. (2000). Psicoterapia breve dinâmica com pacientes borderline: uma proposta viável. São Paulo: Casa do Psicólogo.

Sampaio, M. I. C., \& Peixoto, M. L. (2000). Periódicos brasileiros de psicologia: indexados nas bases de dados LILACS e PsycINFO. Boletim de Psicologia, 50(112), 65-73.

Schoueri, P. C. L., \& Segre, C. D. (1999). Conceito de psicoterapia dinâmica breve (P.D.B). Jornal Brasileiro de Psiquiatria, 48(1), 5-8.

Segre, C. D. (Org.). (1997). Psicoterapias breves. São Paulo: Lemos.

Sifneos, P. E. (1972). Short-term psychotherapy and emotional crisis. Cambridge: Harvard University Press.

Sifneos, P. E. (1984). The current status of individual short-term dynamic psychotherapy and its future: an overview. The Americam Journal of Psychiatry, 38(4), 471-483.

Sifneos, P. E. (1989). Psicoterapia dinâmica breve: avaliação e técnica (A. E. Fillmann, Trad.). Porto Alegre: Artes Médica. (Texto original publicado em 1979)

Yamamoto, O. H., Souza, C. C., \& Yamamoto, M. E. (1999). A produção científica na Psicologia: uma análise dos periódicos brasileiros no período de 1990-1997. Psicologia: Reflexão e Crítica, 12(2), 549-565.

Yamamoto, O. H., Lo Bianco, A. C., Hutz, C. S., Bueno, J. L. O, Guedes, M. C., Koller, S.H., \& Menandro, P. R. N. (2002). Avaliação de periódicos científicos brasileiros da área da psicologia. Ciência da Informação, 31(2), 163-177.

Yoshida, E. M. P., (1990). Psicoterapias psicodinâmicas breves e critérios psicodiagnósticos. São Paulo: EPU.

Yoshida, E. M. P., \& Enéas, M. L. E. (Orgs.). (2004). Psicoterapias psicodinâmicas breves: propostas atuais. Campinas: Alínea.

Neci Sena Ferreira, psicóloga e mestre em Psicologia Clínica pela Pontifícia Universidade Católica de Campinas, é professora na Universidade Federal do Tocantins. Endereço para correspondência: SQN 114, B1. G, Ap. 204; Brasília, DF; CEP 70764-070. Tel.: (61) 349-8051. E-mail: necisena @ terra.com.br

Elisa Medici Pizão Yoshida, doutora em Psicologia Clínica pela Universidade de São Paulo, é professora no Programa de Pós Graduação em Psicologia da Pontifícia Universidade Católica de Campinas, SP.

E-mail: eyoshida.tln@terra.com.br 